\title{
Allergic contact dermatitis caused by diclofenac cream
}

\author{
Carrie B. Lynde MD, Tadeusz A. Pierscianowski MD, Melanie D. Pratt MD
}

Previously published at www.cmaj.ca

$\mathrm{A}$ 40-year-old man presented with a severe rash on his left forearm. One week earlier, he had experienced tendonitis in his left elbow and had treated it with diclofenac cream, a topical nonsteroidal anti-inflammatory drug (NSAID), applied four times daily. The area had become slightly erythematous three days later and then rapidly developed blistering on the fourth and fifth days. The eruption was intensely itchy and spread from the elbow to the wrist. Although the patient had noted some improvement in the tendonitis, he stopped using the diclofenac cream on the fifth day because of the rash.

The patient's medical history included Crohn disease. He was not taking medications and had no known allergies. He reported that he had used the diclofenac cream one year earlier for tendonitis. At that time, a mild erythema had developed on his left forearm after one week of application of the cream and had resolved on its own.

Examination revealed an extensive, poorly demarcated, erythematous and scaly plaque on the left extensor forearm with vesicles and yellow crusting. The affected area extended from the elbow to just above the wrist and corresponded to the area of application of the diclofenac cream (Figure 1).

We diagnosed contact dermatitis caused by allergy to the diclofenac cream with possible impetiginization. The patient may have been sensitized by treatment with the cream the previous year. He was instructed to stop using diclofenac cream and given oral clarithromycin $250 \mathrm{mg}$ twice daily for five days and fluocinonide $0.05 \%$ cream topically twice daily.

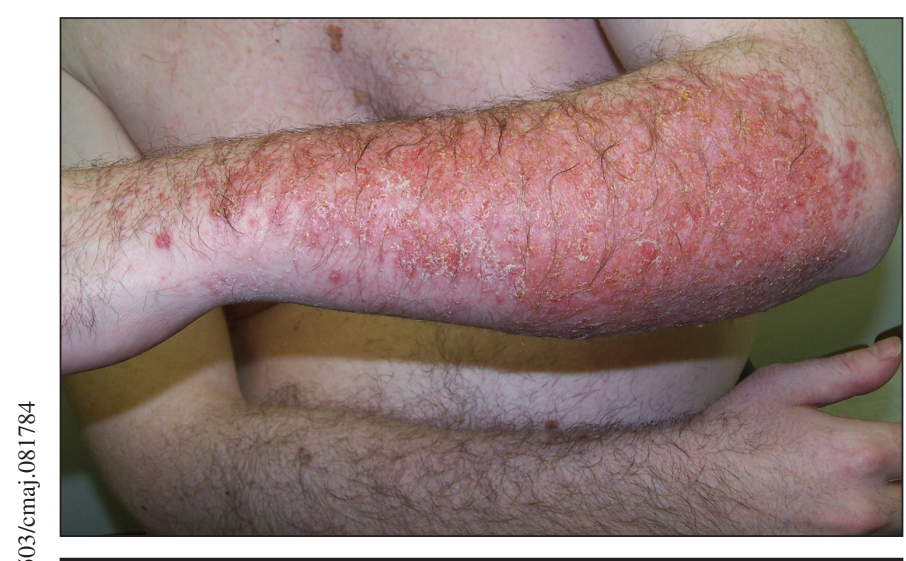

Figure 1: Allergic contact dermatitis on the forearm of a 40year-old man after the use of diclofenac cream.

\section{Key points}

- Allergic contact dermatitis should be suspected when a rash corresponds to the location of contact with a potentially sensitizing substance.

- Known to cause allergic contact dermatitis, propylene glycol is a ubiquitous substance found in many topical medications, including corticosteroid creams.

- If dermal allergy to propylene glycol is suspected, the topical agent should be stopped and replacement with an agent free of propylene glycol should be considered.

Although the patient noted an improvement in the rash with use of the fluocinonide $0.05 \%$ cream, the dermatitis worsened after one week. The fluocinonide $0.05 \%$ cream was discontinued and a course of oral prednisone $40 \mathrm{mg}$ daily was prescribed. The dosage of prednisone was tapered over three weeks, resulting in improvement in the dermatitis.

A patch test was performed using the standard screening series of 65 allergens developed by the North American Contact Dermatitis Group, as well as the patient's own diclofenac cream, fluocinonide $0.05 \%$ cream and selected additional allergens. Diluted allergens in Finn chambers were placed on uninvolved skin on the patient's back and held in place using nonallergenic tape. The patient was instructed to avoid activity that could disturb the chambers, such as bathing or exercise. The Finn chambers and tape were removed at 48 hours, and a reading was performed at 96 hours. Positive skin reactions to propylene glycol (3+) and to the patient's own diclofenac cream $(3+)$ were found. Positive results were found for other agents in the patch test as well but were not relevant to the dermatitis.

\section{Discussion}

Diclofenac cream contains propylene glycol, diclofenac, dimethyl sulfoxide, ethanol and glycerin. Patch-testing in our patient showed that the offending agent was not the NSAID, but rather propylene glycol, which is present in the vehicle of

From the Division of Dermatology (Lynde), University of Toronto, Toronto, Ont.; and the Division of Dermatology (Pierscianowski, Pratt), Department of Medicine, University of Ottawa, Ottawa, Ont.

Cite as CMAJ 2009. DOI:10.1503/cmaj.081784 
Box 1: Examples of sources of exposure to propylene glycol

- Emollients (e.g., moisturizing lotions and creams, cosmetics)

- Industrial agents (e.g., automotive solvents, plasticizers, resins)

- Tobacco products

- Certain foods and beverages (e.g., some cake mixes, whipped topping mixes, microwave popcorn, prepared cakes and carbonated drinks)

- Personal hygiene products (e.g., shaving cream)

- Topical medications (e.g., acne preparations, antibiotic and antifungal ointments, corticosteroid creams)

- Electrode gels

- Contraceptive gels

the cream. Fluocinonide $0.05 \%$ cream also contains propylene glycol, hence the worsening of our patient's dermatitis.

Propylene glycol (Propan-1,2-diol, E1520) is a colourless, viscous and virtually odourless dihydric alcohol with a very low toxicity. Considered an excellent carrier and solvent, propylene glycol is used widely in emollient creams, lotions and personal hygiene products because it easily penetrates the skin. As a vehicle, it is found in many topical, oral and injectable pharmaceutical preparations and in some ultrasound gels and electrocardiogram electrodes. It is also used as a preservative in food and as a humectant food additive. In industry, it is used as an intermediate for the synthesis of other chemicals, as a solvent and as a plasticizer. Cutaneous exposure to propylene glycol usually occurs through contact with cosmetics or topical medications. Some examples of common sources of exposure to propylene glycol are listed in Box 1.

Our patient's case shows the implications of the ubiquitous use of propylene glycol. The patient was prescribed fluocinonide $0.05 \%$ cream to treat his allergic contact dermatitis. Like many commonly used corticosteroid creams, fluocinonide $0.05 \%$ cream contains propylene glycol, which explains why the patient's dermatitis worsened rather than improved when the cream was applied. The patient did not have a positive patch-test result for the fluocinonide cream $0.05 \%$, however, despite the propylene glycol content. Reproducing a positive reaction to the offending agent in a patch test can be difficult, particularly when the test involves a corticosteroid cream with anti-inflammatory properties.

If contact dermatitis with allergy to propylene glycol is suspected, the offending agent should be discontinued and the prescription of a corticosteroid ointment or cream without propylene glycol should be considered. Betamethasone valerate $0.1 \%$ ointment, for example, or a noncorticosteroid ointment such as tacrolimus $1 \%$ ointment, can be used safely. While awaiting identification through patch testing of the culpable allergen, the patient should be advised to use products that are free of propylene glycol.

Diclofenac is a widely used NSAID derivative of phenylacetic acid and is used in oral, topical and parenteral preparations. Although not the allergic constituent in our patient's case, diclofenac has been implicated in allergic contact reac- tions to rheumatic anti-inflammatory gel and eyedrops. ${ }^{1}$ Photoallergic contact reactions have been reported with diclofenac gel used as a topical treatment for actinic keratoses. ${ }^{2}$ Topical diclofenac has been reported to cross-react with topical acelofenac. ${ }^{3}$

\section{Systemic contact dermatitis}

Cutaneous sensitization, as in cases of allergic contact dermatitis, has been associated with systemic contact dermatitis. In the latter condition, systemic exposure to a chemical (i.e., oral, inhalant or intravenous) in a patient who has been previously cutaneously sensitized results in a widespread symmetric dermatitis or recall dermatitis localized to the area of previous sensitization. ${ }^{4}$

Patch testing showed that our patient was not allergic to diclofenac, but to its vehicle, propylene glycol. Once this allergy was identified, systemic diclofenac could be safely ingested. Systemic contact dermatitis has been reported with ingestion of food containing propylene glycol after contact sensitization; experts therefore recommend advising patients of this possibility. ${ }^{5}$

\section{This article has been peer reviewed.}

\section{Competing interests: None declared.}

Contributors: Carrie Lynde and Tadeusz Pierscianowski were involved in the conception of the article and drafting of the manuscript. All of the authors were involved in the analysis and interpretation of the data and critical revision of the manuscript for important intellectual content. All of them approved the final version submitted for publication.

\section{REFERENCES}

1. Kleyn CE, Bharati A, King CM. Contact dermatitis from 3 different allergens in Solaraze gel. Contact Dermatitis 2004;51:215-6.

2. Kowalzick L, Ziegler H. Photoalleric contact dermatitis from topical diclofenac in Solaraze gel. Contact Dermatitis 2006;54:348-9.

3. Pitarch Bort G, de la Cuadra Oyanguren J, Torrijos Aguilar A, et al. Allergic contact dermatitis due to aceclofenac. Contact Dermatitis 2006;55:365-6.

4. Jacob SE, Zapolanski T. Systemic contact dermatitis. Dermatitis 2008;19:9-15.

5. Lowther A, McCormick T, Nedorost S. Systemic contact dermatitis from propylene glycol. Dermatitis 2008;19:105-8.

Correspondence to: Dr. Carrie B. Lynde, clo Dr. T. Pierscianowski, Hunt Club Medical Centre, 103-3328 McCarthy Rd., Ottawa ON K1V 0H9; fax 613 738-5328; carrie.lynde@ utoronto.ca

The section Cases presents brief case reports that convey clear, practical lessons. Preference is given to common presentations of important rare conditions, and important unusual presentations of common problems. Articles start with a case presentation (500 words maximum), and a discussion of the underlying condition follows (1000 words maximum). Generally, up to 5 references are permitted and visual elements (e.g., tables of the differential diagnosis, clinical features or diagnostic approach) are encouraged. Written consent from patients for publication of their story is a necessity and should accompany submissions. See information for authors at www.cmaj.ca 